\title{
Resonant Infra-red Pulsed Laser Ablation of Polymers with single picosecond pulses generated by an optical parametric amplifier
}

\author{
Malte Duering $^{1}$, Richard Haglund ${ }^{2}$ and Barry Luther-Davies ${ }^{1}$ \\ ${ }^{1}$ Laser Physics Centre, Research School of Physics and Engineering, The Australian \\ National University, Canberra ACT 2600 Australia. ${ }^{2}$ Department of Physics and \\ Astronomy, Vanderbilt University, Nashville, TN, USA. \\ barry.luther-davies@anu.edu.au
}

\begin{abstract}
We have used a mid-IR optical parametric amplifier for single pulse ablation of polystyrene via resonant infra-red pulsed laser ablation. C2010 Optical Society of America

OCIS Codes 350.3390, 310.1860
\end{abstract}

Introduction.

Resonant infra-red pulsed laser ablation (RIR-PLA) [1] has been reported to allow the vaporization of technologically important polymers and deposition of polymer films without scissoring of the polymer chains which occurs using conventional pulsed laser ablation with UV lasers. RIR-PLA uses a laser source tuned to one of the vibrational bands of the polymer in the infra-red. For materials containing C-H bonds wavelengths in the 3-4 $\mu \mathrm{m}$ are required. So far most studies of RIR-PLA has been carried out using a free electron laser (FEL) at Vanderbilt University. However the technique is unlikely to find widespread application until bench-top laser sources are developed.

Here we describe RIR-PLA using a tuneable mid-IR source based on optical parametric amplification in periodically poled lithium niobate $(\mathrm{PPLN})$ that produces single $\approx 8$ ps duration pulses with and energy of up to $170 \mu \mathrm{J}$. We used these pulses to study single pulse ablation of polystyrene excited at an aromatic resonance around $3.26 \mu \mathrm{m}$, determining the ablation threshold and morphology of the ablated region.

\section{Experiments}

Based on data from the FEL studies, we expect the ablation threshold for single picosecond duration pulses to lie in the range of a few hundred $\mathrm{mJ} / \mathrm{cm}^{2}$. To produce such fluences from single, picosecond pulse the pulse energy $>100 \mu \mathrm{J}$ is required in a near diffraction-limited beam. We generated such pulses using an PPLN optical parametric amplifier (OPA) [2] pumped by $\approx 15 \mathrm{ps}$ duration pulses at $1053 \mathrm{~nm}$ from a Nd:YLF master oscillator power amplifier (MOPA). The MOPA used a flashlamp-pumped regenerative amplifier to produce $1.5 \mathrm{~mJ}$ pulses at $1064 \mathrm{~nm}$ at repetition rates up to $20 \mathrm{~Hz}$. These pulses pumped a two-stage optical parametric amplifier seeded by a $300 \mathrm{~mW} \mathrm{CW}$ beam from a tuneable semiconductor diode laser combined with an erbium doped fiber amplifier (EDFA). Amplification of the seed led to creation of a tuneable idler output in the 3200-3380nm range limited by the bandwidth of the EDFA. The two-stage OPA provided an overall conversion efficiency of about $35 \%$ (to signal and idler output combined); $55 \%$ pulse shortening and yielded $\approx 170 \mu \mathrm{J}$ single pulses at $3.3 \mu \mathrm{m}$. After separation of idler from the pump and signal beams about $100 \mu \mathrm{J}$ could be delivered to the sample through a $75 \mathrm{~mm}$ focal length $\mathrm{CaF}_{2}$ lens. The output beam from the OPA was near diffraction-limited and was focussed to a spot $\approx 70 \mu \mathrm{m}$ in diameter on sample of polystyrene in air to a maximum fluence $\approx 2 \mathrm{~J} / \mathrm{cm}^{2}$.
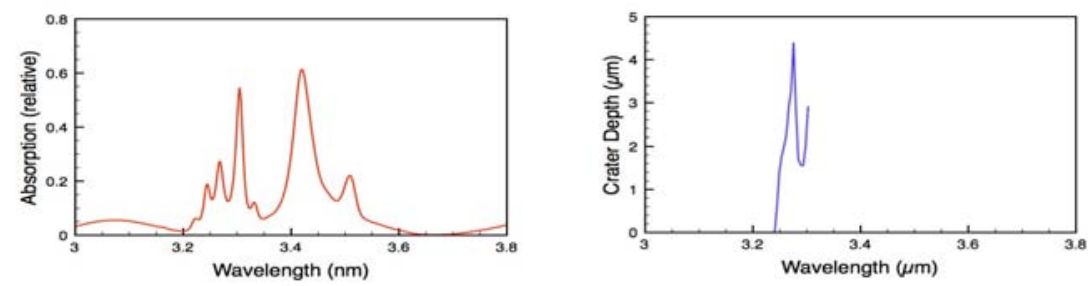

Fig. 1. LHS: 1(a) absorption spectrum of polystyrene; RHS: 1(b) Data on crater depth vs wavelength used to tune OPA optimally to the absorption line. 


\section{CMH3.pdf}

The idler was tuned to $\approx 3.26 \mu \mathrm{m}$ which corresponded to one of the aromatic resonances in polystyrene (Fig. 1a). The depth of the crater produced in the surface of the sample was measured using an optical profiler as a function of the output wavelength from the OPA to fine tune the system to the peak of the resonance (Fig. 1b). An image of a crater formed at about twice the ablation threshold is shown in Fig. 2 and consists of a region with a sharp boundary within which the polymer swells about 600nm above the original sample surface, and an internal crater about $2 \mu \mathrm{m}$ deep in these conditions from which polymer has been ablated. Below the ablation threshold only swelling of the polymer occurred. Apparent from Fig. 2 is the complete absence of ejection of any molten material from the crater. The sidewalls are exceptionally smooth with no evidence of droplets contaminating the surrounding surface of the target.

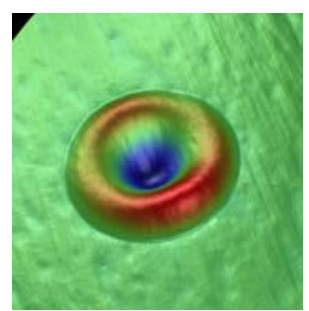

Fig. 2. Image of a crater in polystyrene at twice the ablation threshold showing both swelling of the material and ablation.

The variation of crater depth was mapped as a function of laser power as shown in Fig. 3 along with a model assuming the material removed corresponded to that where the deposited energy density exceeded a threshold value with its penetration into the material decaying exponentially which qualitatively aggress with the data and is consistent with an ablation threshold of about $0.3 \mathrm{~J} / \mathrm{cm}^{2}$.

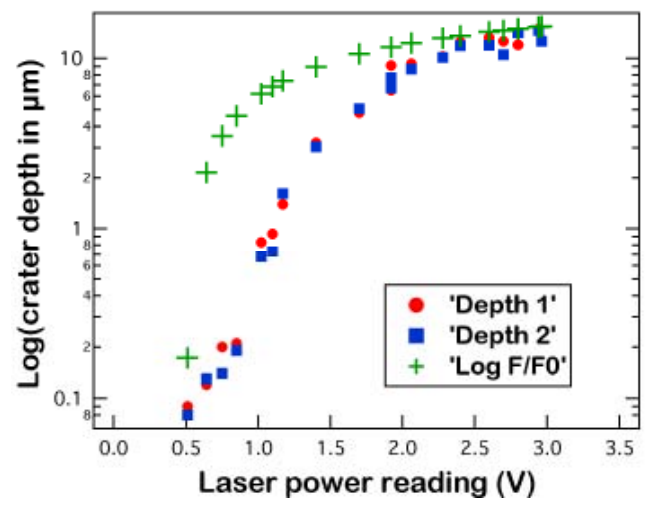

\section{Conclusions}

We have shown that strong ablation of polystyrene can be achieved using single picosecond pulses via resonant infrared pulsed laser ablation using an OPA. This demonstrates that RIR-PLA of polymers is achievable with a bench-top sources based on high repetition rate psec industrial Nd:YAG lasers opening up the possibility that RIR-PLA can be used to deposit technologically important polymer films from the vapour phase.

\section{Acknowledgements}

The support of the Australian Research Council is gratefully acknowledged.

[1] D. M. Bubb, J. S. Horwitz, J. H. Callahan, R. A. McGill, E. J. Houser, D. B. Chrisey, M. R. Papantonakis, R. F. Haglund, M. C. Galicia, and A. Vertes, Resonant infrared pulsed-laser deposition of polymer films using a free-electron laser, Journal Of Vacuum Science \& Technology A 19(5), 2698-2702 (2001).

[2] V. Z. Kolev, M. W. Duering, B. Luther-Davies, A. V. Rode, Compact high power optical source for resonant infrared pulsed laser ablation and deposition of polymer materials, Optics Express 14, 1230212309 (2006). 